\title{
Teaching Fundamental Programming Using Augmented Reality
}

\author{
https://doi.org/10.3991/ijim.v13i07.10738 \\ Salin Boonbrahm ${ }^{(凶)}$, Poonpong Boonbrahm, Charlee Kaewrat, Prasert Pengkaew, \\ Prathomjit Khachorncharoenkul \\ Walailak University, Nakhon Si Thammarat, Thailand \\ Salil.boonbrahmegmail.com
}

\begin{abstract}
To learn a programming language, the students have to understand the logical flow of the commands as well as the syntax. The logical flow might be more difficult to understand when compared with a syntax which can detect easily. The primary flow of commands or the control structures includes the sequence, condition or selection, and iteration. The students construct the program flowchart by using these control structure. They also have to understand the result of each command execution, step by step. In this research, we propose the technique for developing the learning tool (AR flowchart) to simulate the result of the commands in program flowchart by using augmented reality (AR), so the learners can visualize the result. With this tool, the students can construct a program flowchart as a series of commands by using AR markers. The result of the execution of these commands can be displayed so the students can see whether the logic of the program is correct or not. The design of this tool aims at increasing student engagement and helping them to understand program logic better. The evaluation of the concept results by the group of university students supports our propose.
\end{abstract}

Keywords - Augmented reality, learning tool, program fundamental teaching, control structure, flowchart

\section{Introduction}

Computer programming may be easy for someone, but lots of students find it is difficult to accomplish, especially for non-science student. The concept of programming relies on the understanding of programming logic. To teach program logic without having to worry about the syntax, program flowchart is introduced. Program flowcharts consist of a set of symbols connect to form the series of commands for the computer. After drawing program flowcharts, the students should check the correctness of the diagram. Since there are no tools available, students have to do deck checking by themselves to get the result. However, for a beginner, it is still hard to understand without visualization of the result. Augmented reality (AR) is used to provide visualization. AR flowchart is designed and developed to help the students to better understand programming logic by delivering the result of each command that the student can see. 
Augmented reality is a technology that provides us with merging the real environment with digital data. There are two types of AR, i.e., marker-based AR and markerless AR. Marker-based AR uses a camera to identify visual markers or objects, to showcase an overlay only when the device detects the marker but marker-less AR relies on a GPS or digital compass, to provide data about the location and the AR visualizations are activated based on these inputs. In this study, the marker-based AR is used, since we want the system to identify each command that the student put into a flowchart by hand. In our design, the symbols in flowchart such as start/end, decision and process will be represented by markers. By aligning the notation (with information) or command in the form of a flowchart, the AR system can read the first symbol (marker) then forward the function or parameter to the next symbol and so on, just like reading each line of the program. After finish reading all of the symbols (markers), then the system compile and generate the result in the form of visualization. Using this technique, students can understand the logical concept of programming logic easily and also enjoy doing them.

\section{$2 \quad$ Related Works}

The applications of AR were developed in many areas including advertising and marketing, architecture and construction, military, and travel [1]. The examples of using AR in marketing are 3D catalogs where customers can try out the merchandise and postal box with different displays during the holiday seasons such as Christmas decoration [2]. AR applications are used in military training and war to enhance the ability of the commander in different situations such as the Battlefield Augmented Reality System (BARS) [3]. The examples when applying AR in the travel or tourism industry are $\mathrm{AR}$ travel guides and $\mathrm{AR}$ museum where additional information can be provided for tourists [4].

There were also many studies that focus on the use of AR in education. In these studies indicates that there are many benefits from using AR such as student outcome improvement [5], enjoyment enhancement, learning motivation enhancement, a better understanding in learning concepts, positive attitude providing, collaboration opportunities providing, and promote self-learning [6]. AR also provides a better tool in prototype development. Students can use AR technology to display digital architecture in the real-world environment without having to build physical construction [5]. With the 3D model, AR will help the students when they are learning of orthographic view [7]. The uses of AR in education are in different approaches such as book, game, discoverybased learning, object modeling, and training $[1,8]$. The areas that AR was applied were several include medical education, chemistry, mathematics, biology, physics, and history [9].

To develop the AR application for education, the learning experience should be focused. The factors that are related to learning experience are those about hardware, software, and content. AR can be applied to different types of display device such as the head-mounted display (HMD), handheld device, overhead projectors, and PC monitor [10] Santos et al., 2014. 
There are several models to design the instructional tool. The ARCS model is used in this study. The ARCS model is a model that focuses on the motivation of the learner. It is believed that to success in learning, the learner should have the motivation to learn first. The model consists of 4 factors including attention, relevance, confidence, and satisfaction [11]. There is also research which emphasis on using AR to replace the real learning experience that student gained from experimenting in the laboratory using realtime interaction of marker-marker interaction [12].

The attention strategy, for this research, aims at how to get the student attended, and the second factor, the relevance is based on the relationship of the instructional tool and the conceptual understanding. The third strategy is confidence that the students can get when applying the different amount of effort to get the correct result while the final policy is satisfaction where the students can see the success and can help the others.

\section{Problem Definition and Requirement Analysis}

Programming is the way to create a set of command to do some activities to get the result or to solve the given problem. When the students start to learn how to program, they should understand basic concepts first. One of those concepts is control structures that include sequence, condition, and iteration [13]. The learners got the tasks or problems that they should create a set of commands using the control structure to achieve it. Without worrying about programming language syntax, program flowchart is the graphical presentation that used to represent the sequence of the commands. The difficulty of using the program flowchart is that the students have to understand how each command works and what will be the result when running commands on the flow. If the result is incorrect, the students have to fix the use of commands in the flowchart. Again, they have to check after executing the commands, whether the result is correct or not. For some students, this may be a difficult task because they cannot see the result. If the students have a tool that they can visualize the result of the command, they can understand how commands on the flowchart work better.

To design and develop a tool to help the students to understand better how the flowcharts work, we can divide it into four components, i.e., concept, content, software, and hardware.

\subsection{Concept}

The idea of teaching fundamental programming where the students can visualize the output from the flowchart diagram can be done using interactive AR. With this concept, each flowchart notation can be replaced by the AR marker and flow of process can be visualized by using marker-marker interaction. Since we can implant the functions or data into AR markers, then programming these markers to interact with others, the final output of the flowchart can be visualized using AR technology. Imagine that we have three markers, A, B and C, placed in respective order. Marker A is set to be the input data, marker $\mathrm{B}$ as the function and marker $\mathrm{C}$ as the output; These can be shown as the equation (1). 


$$
y=f(x)
$$

From Equation 1, $\mathrm{x}$ is marker $\mathrm{A}$ or the input; $\mathrm{f}$ is marker $\mathrm{B}$ which is the function or operation, and $\mathrm{y}$ is marker $\mathrm{C}$ which is output. The example of the AR marker-marker interaction shown in Figure 1 is the operation of the logic gate. From this figure, the input is zero, and the function is "NOT" operator, then the output from the operation is equal to 1 .

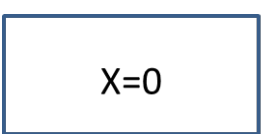

Input data

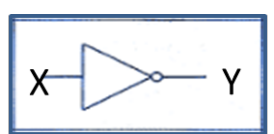

function

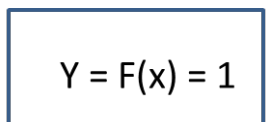

output

Fig. 1. Marker-marker interaction for NOT gate

When implementing Figure 1 with the real markers (Figure 2(a)), the input or Marker $A$ is zero or off, Marker B indicates NOT operator, and the result of the interaction between Marker A and B will show on Marker C. When running the program, the simulation is shown in Figure 2(b). The same technique will be used in simulating the flowchart diagram.

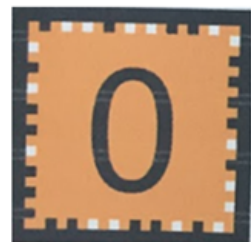

Marker A

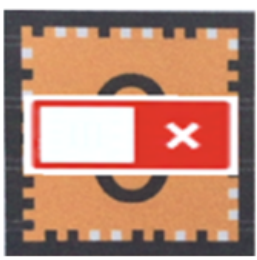

Marker A

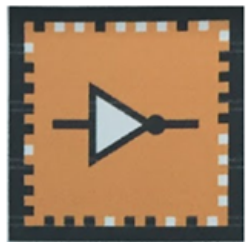

Marker B

(a)

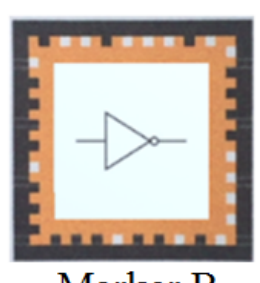

Marker B

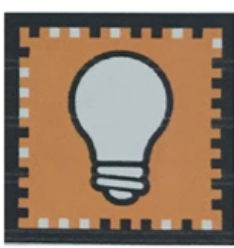

Marker C

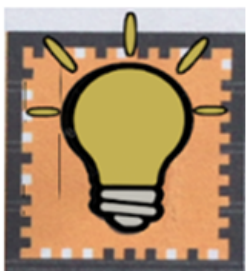

Marker C

(b)

Fig. 2. Implementation of the marker-marker interaction in logic gate (a) and the visual simulation (b) 


\subsection{Content}

Content will cover the problem that students have to solve and the solution that the students have to find out. To learn programming skill, the students have to practice solving many problems, so some different problems should be able to set when using this tool. They must understand the problem first, and then design the logic or sequence of commands to solve the problem which means that the students can set different problems based on this tool.

Building a flowchart to solve the problem is like setting the flow of command. Students should be able to show that they understand types of command as well as the order of command in the flowchart. The primary control structures that included are sequence, condition and repetition or loop as shown in Figure 3. These structures will be used to construct a series of command to provide the result for the problem. Moreover, the tool has to support the use of variable such as they may require setting the initial values for the variables and setting Boolean expression for the condition.

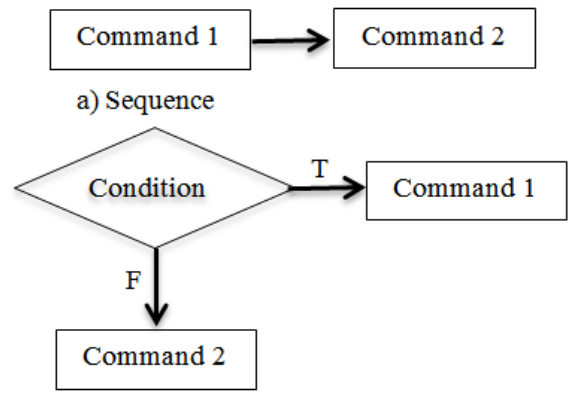

b) Condition

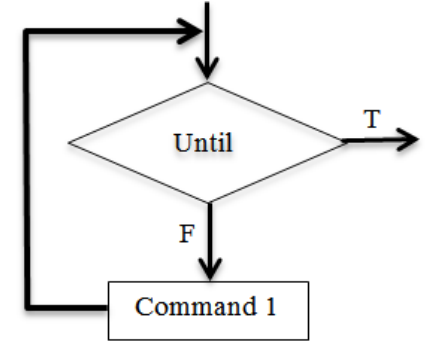

c) Loop

Fig. 3. Basic control structures

\subsection{Software}

The software application is developed to capture the image of the flowchart that student construct, process the program and show the result of command execution. From the capturing image, it should be able to identify the command, the value of the variable and Boolean operator. Then the software should simulate the result of each command. Since the commands are connected, the output from the previous command will be sent to be the input of the next command as mentioned in 3.1. The user interface of the application should provide enough information for the students to understand what to do, or what is the result.

In this research, the software required for developing the AR applications consists of Unity 3D cross-platform game engine along with Vulforia AR Software Development Kit (SDK) for mobile. We also used Xcode, which is an integrated development environment (IDE) for macOS, for building applications file for iOS devices. 


\subsection{Hardware}

There are two groups of hardware used, one for generating AR applications and the second one for running the applications and visualize the output. For developing applications program, MacBook Pro with Intel core i7, 16 GB memory and Intel Iris Plus Graphics 650, was used along with Logitech Brio. For running the applications, the device should be able to use as an input device to track the commands in a flowchart. It is also used as an output device to display the digital image over the real world object. The output is display in real time, so it should be able to display smoothly. In this case, iOS devices, such as iPads or iPhone was used for this purpose.

\section{The Design of AR Flowchart System}

The theme for setting the problem, in this case, is the parcel delivery. The reason for selecting this theme is that the objective of the problem is clear to everyone. The delivery person has to send the right parcel to the right destination. We can also add more condition when the learner understands the basic one such as when there is nobody at the destination the delivery person has to take the parcel back to the office. The board in Figure 4 is showing the map of the road that used as the main ground for the experiment. The environment of the roadmap can easily set by placing houses on a different position on this map.

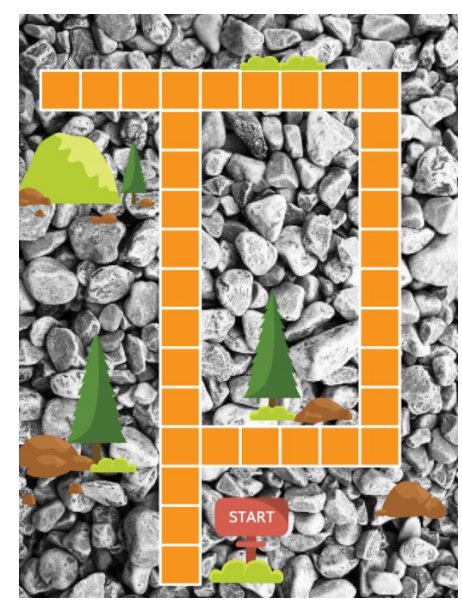

Fig. 4. Roadmap

The commands for this case are commands for delivery the parcel and commands that give the direction to the delivery truck such as go straight, turn left, turn right, and U-turn. There are many variables used for this problem domain such as the color of the house or absentee of the receiver. For example, the delivery person has to send the parcel to the house with the same color, i.e., he or she has to send the red parcel to the 
red house. Another condition for parcel delivery is that the delivery person cannot deliver the parcel to the empty house.

Figure 5 shows the example of the markers which are the blue house destination, the receiver, the delivery truck, go straight command, and the blue parcel. With these markers, the students can construct the flowchart that contained a series of the marker. The students can also create the condition statement by setting the condition of the color of the parcel that has to be matching the color of the house at the destination.
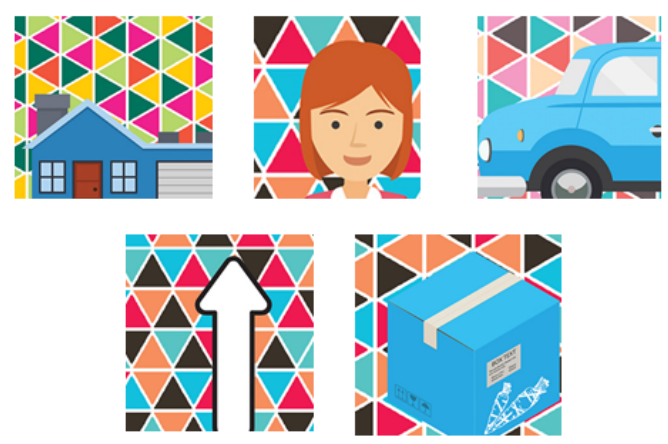

Fig. 5. The Markers

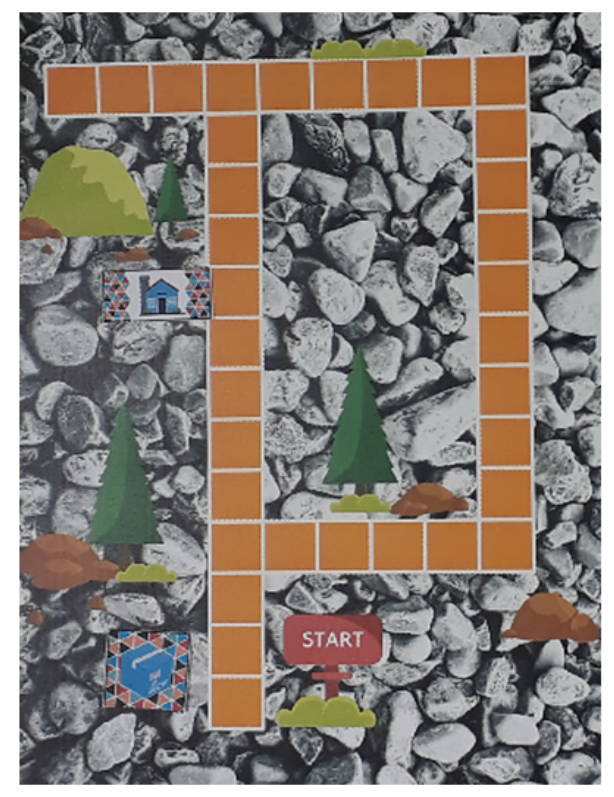

(a)

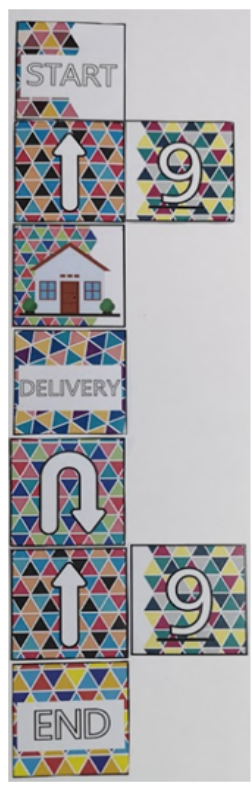

(b)

Fig. 6. A simple delivery problem and the solution 
For example, if the problem is "give the instruction (to the delivery person) to send the parcel to the blue house," the roadmap should contain two markers that indicate the parcel and the destination as shown in Figure 6 (a). The learner is then constructing the flow of instruction as a solution by using the set of markers as shown in Figure 6 (b). Figure 6 can represent question and answer or solution to that question. The problem can be changed differently by moving the markers on the roadmap.

The flowchart should be constructing in a top-down approach. With the house marker, students can set their roadmap environment by placing the house marker on this map. The AR flowchart software is designed to detect the new environment of the roadmap, the series of command and variable in the flowchart, and the input (color of the parcel to deliver). The red or green color is used to indicate the completeness of marking tracking process as shown in Figure 7. Besides the use of color, the learner can also check the integrity from the list of markers that have been scanned showing on the left-hand side as shown in Figure 7 (b).

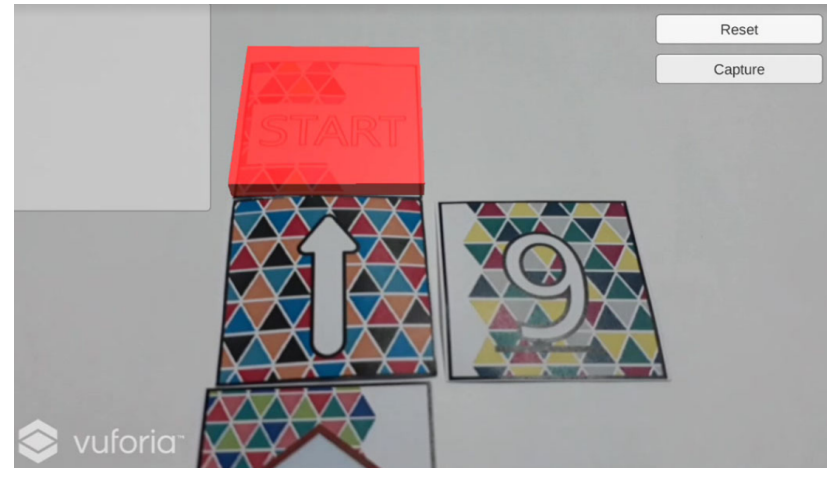

(a)

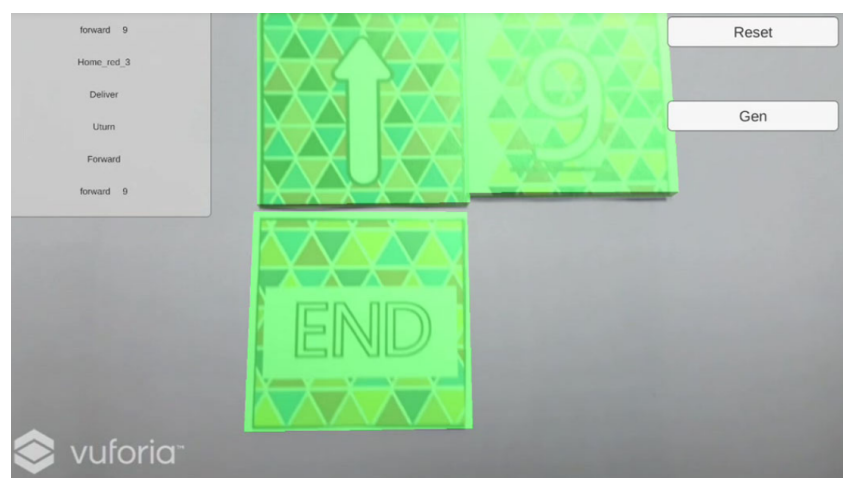

(b)

Fig. 7. Tracking the markers in a flowchart 
For the hardware factor, Apples' iPad has been used as a handheld device to capture the marker images and show the result of the command execution. From Figure 8(a), the $3 \mathrm{D}$ animated picture of the truck with parcel will display after the capturing processes of the roadmap environment, the flowchart, and the delivery parcel are completed. Figure 8(b) shows the truck on the move according to the command after the Start Button is selected. Figure 8(c), (d), and (e) shows the process of delivery and the truck returns to the starting point.

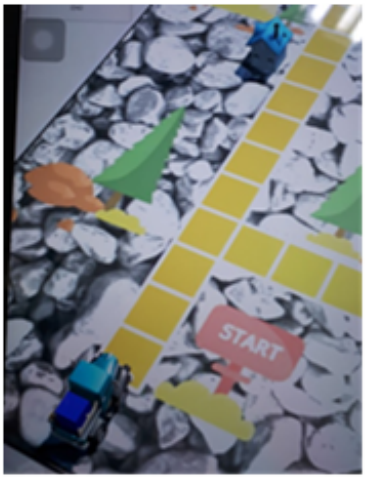

(a)

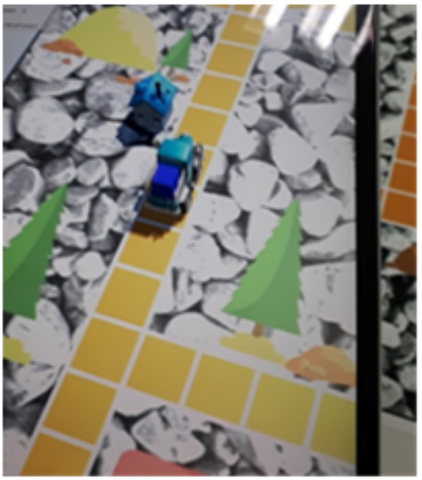

(b)

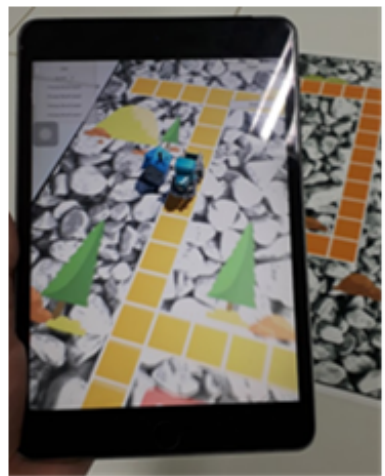

(c)

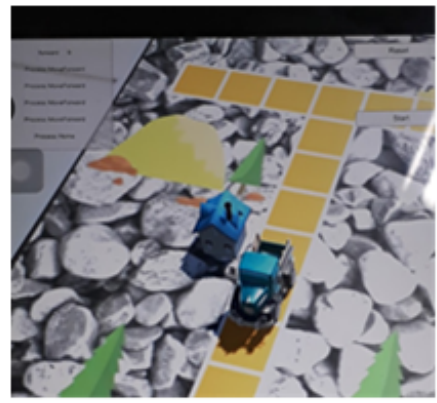

(d)

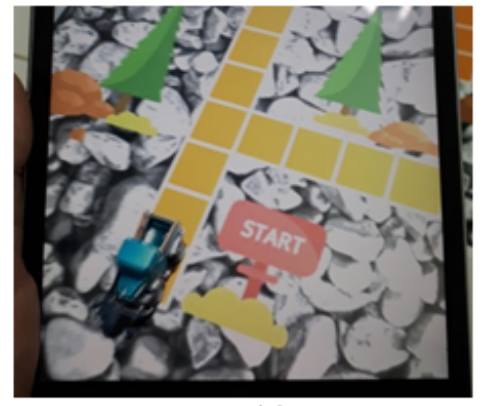

(e)

Fig. 8. The visualization showing the result from commands in flowchart

\section{Evaluation}

The experiment was conducted at Walailak University (Figure 9). The sample size for this experiment was 20 . The participants were students who take the study program that offering Programming Language course from School of Informatics and School of Science. They were the students in Information Technology (IT), Software Engineering (SWE), Computational Science (CSC), and Multimedia Technology and Animation (MTA) study program as shown in Table 1. 


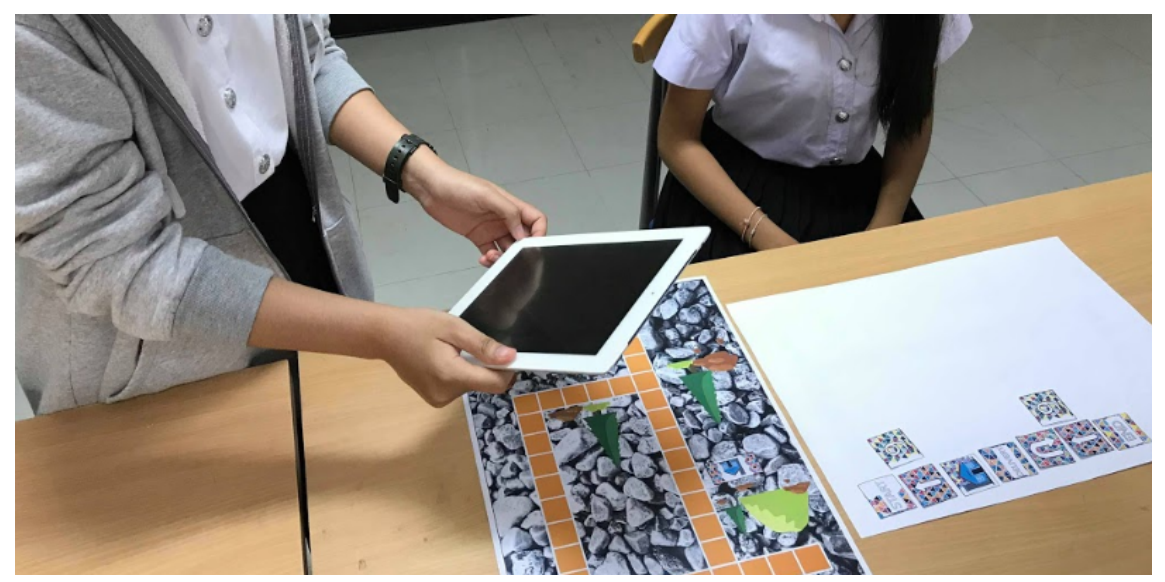

Fig. 9. Students are experiencing the AR flowchart

Table 1. Number of student from the study program

\begin{tabular}{|l|c|c|}
\hline \multicolumn{1}{|c|}{ Study program } & Number & Per cent \\
\hline IT & 10 & 50 \\
\hline SWE & 2 & 10 \\
\hline CSC & 3 & 15 \\
\hline MTA & 5 & 25 \\
\hline
\end{tabular}

The experiment starting from setting both simple and complex problems that students have to find the results such as how to deliver the parcel to the destination with the condition that the parcel can only deliver if there is the receiver or person at the destination. Next, explaining the meaning of the markers and show them how to connect the makers to form the flowchart. After the flowchart was constructed, we showed them how the application on the handheld device works and how they can see the result. Then we set the new task or problem and ask each student to construct a new flowchart that can give the result for that task. When the students finished the test, they were asked to answer the questionnaire that consists of six close-ended questions and one open-end question. The Likert scale was used to answer the closed end questions. The highest score is five, and the lowest score is one.

\section{$6 \quad$ Result and Discussions}

Table 2. Response from closed-end questions

\begin{tabular}{|l|c|c|}
\hline \multicolumn{1}{|c|}{ Question } & Mean & SD \\
\hline 1. The use of the symbol in the flowchart is easy to understand & 4.60 & 0.50 \\
\hline 2. The ease of flowchart construction using the markers & 4.35 & 0.67 \\
\hline 3. The ease of using the application to simulate the result & 4.45 & 0.60 \\
\hline 4. The understandable of the result from the simulation & 4.50 & 0.61 \\
\hline 5. The improvement of understanding the control structure by using the flowchart & 4.70 & 0.57 \\
\hline 6. Overall satisfaction of the user & 4.70 & 0.47 \\
\hline
\end{tabular}


The results from 20 students are summarized in Table 2, and the average scores for each question from the students in different study program are shown in Figure 9. The highest mean value is 4.7 from question 5 and 6 . For the first question, the students found that it is easy to understand the image on each marker. When they were asked about the ease of flowchart construction in question 2, the mean value for this item is 4.35 which indicate that it is easy. The students found that the use of the software application is also easy (mean value is 4.45 ). The visualization showing the result of the command is also easy to understand. It is found that the students were highly agreed that using this AR flowchart will improve their understanding of using control structure. They are very satisfied with using it.

For the open-end question that asks the students to express their opinion, there were 11 responses from 20 students. Some of the opinions are similar; they can be grouped into four categories:

- This project should continue

- Sometimes it is difficult to use the iPad to track the markers

- Wonderful technology

- It is fun

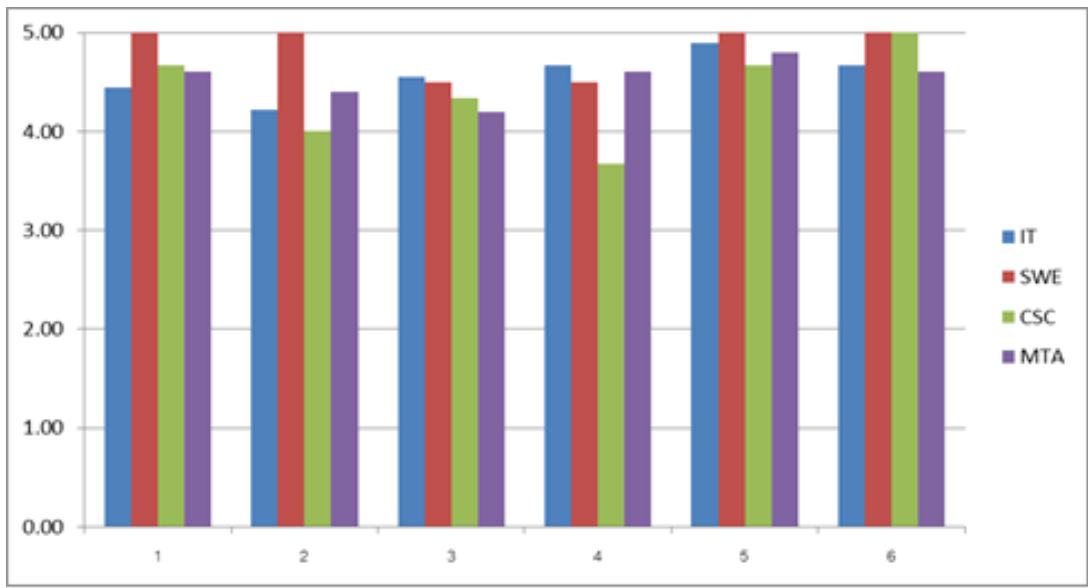

Fig. 10.The response from students in different study program

\section{Conclusion and Future Works}

The AR flowchart can help the students to visualize the result of the command execution. From observation, the students enjoy setting several problems and observe the results. When the result is not correct (i.e., deliver the parcel to the incorrect receiver), they can adjust the diagram and check the result. The AR flowchart can be considered to support the student-centred approach because the student can set the task or problem that they want to solve. AR flowchart also enhances student engagement since the student try to find the set the command that the delivery truck complete the delivery 
mission successfully. When one student had finished the experiment, they did not leave the class but prefer to observe other students' experiment.

The future work for building this AR flowchart will be the focus on upgrading the code on tracking the maker and add the complexity of condition and iteration control structures.

\section{$8 \quad$ References}

[1] Yuen, S. C., Yaoyuneyong, G., \& Johnson, E. (2011). Augmented reality: An overview and five directions for AR in education. Journal of Educational Technology Development and Exchange (JETDE), 4 (1), 119-140. https://doi.org/10.18785/jetde.0401.10

[2] Levski, Y. (2017). 10 Real World Examples of AR Marketing Success. Augmented Reality. https://appreal-vr.com/blog/10-augmented-reality-marketing-examples/

[3] Livingston M.A. et al. (2011). Military Applications of Augmented Reality. In: Furht B. (eds) Handbook of Augmented Reality. Springer, New York, NY.

[4] Kounavis C.D., Kasimati A.E., Zamani, E.D. (2012). Enhancing the Tourism Experience through Mobile Augmented Reality: Challenges and Prospects. International Journal of Engineering Business Management, 4, 1-6. https://doi.org/10.5772/51644

[5] Fernandez, M. (2017). Augmented virtual reality: How to improve education systems. Higher Learning Research Communications, 7(1), 1-15. http://dx.doi.org/10.18870/hlrc. v7i1.373.

[6] Akcayir, M. \& Akcayir, G. (2017). Advantages and challenges associated with augmented reality for education: A systematic review of literature. Educational Research Review, 20, 1-11. https://doi.org/10.1016/j.edurev.2016.11.002

[7] Figueiredo, M., Gomes, J., Gomes, C., \& Lopes J. (2014). Augmented reality tools and learning practice in mobile-learning. Recent Advances in Educational Technologies and Methodologies, Proceedings of the 13th International Conference on Education and Educational Technology (EDU '14), 63-72. https://doi.org/10.4018/978-1-5225-5469-1.ch076

[8] Liarokapis, F. \& Anderson, E.F. (2010). Using augmented reality as a medium to assist teaching in higher education. Eurographics. http://dx.doi.org/10.2312/eged.20101010.

[9] Saidin, N.F., Halim, N.D.A., \& Yahaya, N. (2015). A review of research on augmented reality in education: advantages and applications. International Education Studies, 20(13). https://doi.org/10.5539/ies.v8n13p1.

[10] Santos, M. E.C., Chen, A., Taketomi, T., Yamamoto, G., Miyazaki, J., \& Kato, H. (2014). Augmented Learning Experiences: Survey of Prototyping Design and Evaluation. IEEE Transaction on Learning Technologies, 7(1), 38-56. https://doi.org/10.1109/tlt.2013.37

[11] Keller, J.M. (1987). Development and Use of ARCS Model of Instruction Design. Journal of Instructional Development, 10, 2-10.

[12] Boonbrahm, P., Kaewrat, C. \& Boonbrahm, S. (2016). Interactive Augmented Reality: A New Approach for Collaborative Learning. Lecture Notes in Computer Science, 9753, pp 115-124. https://doi.org/10.1007/978-3-319-39483-1_11

[13] Farrel, J. (2010). Just Enough Programming Logic and Design. Course Technology. 


\section{Authors}

Salin Boonbrahm is a lecturer in Information Technology Department, School of Informatics, Walailak University. She got Ph.D. in Computer Science from University of New South Wales, Australia.

Poonpong Boonbrahm is an Associate Professor and Dean of School of Informatics, Walailak University. He got his Ph.D. in Physics from Kent State University, USA.

Charlee Kaewrat and Prasert Pengkaew are postgraduate students in Ph.D. and Master Programs in Management of Information Technology, School of Informatics, Walailak University.

Prathomjit Khachorncharoenkul is a lecturer in Mathematics Department, School of Science, Walailak University. She got her Ph.D. in Mathematics from Chulalongkorn University, Thailand.

Article submitted 2019-04-26. Resubmitted 2019-06-04. Final acceptance 2019-06-04. Final version published as submitted by the authors. 